\title{
Estudio de estructuras de cis- y trans-estilbeno mediante microscopiade fuerza atómica
}

\author{
Study of cis- and trans-stilbene structures \\ by atomic force microscopy
}

\author{
Sandra Marina Mendoza ${ }^{1}$, Mario César Guillermo Passegg ${ }^{2,3}$ \\ Julio Ferrón ${ }^{2,3}$
}

\footnotetext{
${ }^{1}$ Departamento de Electromecánica, Facultad Regional Reconquista (UTN) - CONICET. Calle 44 No 1000, (3560) Reconquista, Argentina.

e-mail: smendoza@ frrq.utn.edu.ar

${ }^{2}$ Laboratorio de Física de Superficies e Interfaces, Instituto de Física del Litoral (IFIS, CONICET-UNL). Güemes 3450, (3000) Santa Fe, Argentina.

${ }^{3}$ Departamento de Materiales, Facultad de Ingeniería Química (UNL). Santiago del Estero 2829, (3000) Santa Fe, Argentina.

e-mail: mpggih@ifis.santafe-conicet.gov.ar; jferron@ifis.santafe-conicet.gov.ar
}

\section{RESUMEN}

La nanotecnología es un área multidisciplinaria que ha tenido un gran auge en los últimos años, principalmente debido al desarrollo de instrumental que permite "ver", estudiar y manipular la materia a nivel atómico y molecular. El estilbeno y algunos de sus derivados son moléculas orgánicas de origen natural, que presentan una estructura química de interés en la fabricación de nanoestructuras y bloques constituyentes de máquinas moleculares (potencialmente aplicables en tecnología de nanosensores, almacenamiento de datos, liberación controlada de fármacos, entre otros). Sin embargo todavía no han sido ampliamente exploradas como nanomateriales. El interés en este tipo de sistemas reside en sus aplicaciones en medicina, farmacia y biotecnología.

Este trabajo presenta los primeros resultados obtenidos al estudiar formas isoméricas de estilbeno: cis- y trans-estilbeno, líquido y sólido a temperatura ambiente, respectivamente, con el objetivo de explorar sus propiedades, al estar las mismas en contacto con superficies policristalinas de oro (Au/mica) y grafito (HOPG).

Se encontró que la inmovilización de trans-estilbeno sobre una superficie puede modificar el proceso de cristalización de estas moléculas orgánicas, cuya morfología depende del sustrato. Mientras que el transestilbeno forma "clusters" que originan cristales de forma pseudo-romboide sobre HOPG, sobre la superficie de Au/mica forma estructuras dendríticas con ramificaciones en ángulos a $90^{\circ}$.

Por otra parte, se observó que el cis-estilbeno a escala nanométrica forma también "clusters" sobre HOPG, comportándose de manera similar al trans-estilbeno. Un paulatino incremento en la cantidad de cis-estilbeno conlleva al crecimiento de dichos "clusters" y a su coalescencia en una fase amorfa. Por el contrario, la interacción molécula-sustrato de cis-estilbeno sobre Au/mica es más lábil que sobre HOPG y ya no resulta posible observar centros de nucleación o nanoestructuras estables. Un aumento en la cantidad de cis-estilbeno sobre $\mathrm{Au} / \mathrm{mica}$ resulta en una especie de película delgada líquida.

Palabras clave: cis-estilbeno, trans-estilbeno, HOPG, Au/mica, AFM.

\section{ABSTRACT}

Nanotechnology is a multidisciplinary field that emerged in recent years due to the development of tools to "see", study and manipulate matter at atomic and molecular levels. Stilbene and some of its derivatives are organic molecules found in nature, which have chemical structures of interest in nano-fabrication of molecular machine building blocks (with prospective applications in nano-sensors, data storage and drug controlled 
release, among others). However, they have not been extensively explored as nanomaterials. This kind of systems is of interest in medicine, pharmacy and biotechnology.

This work presents the first results obtained by studying stilbene isomers: cis- and trans-stilbene, liquid and solid at room temperature, respectively, to explore their properties on polycrystalline substrates of gold (Au/mica) and graphite (HOPG).

We found that immobilization of trans-stilbene on a substrate can modify the crystallization process of the organic molecules, and the crystal morphology depends on the substrate. While trans-stilbene forms clusters that lead to pseudo-rhomboidal crystals on HOPG, dendritic structures with $90^{\circ}$ proyected branches are formed on equally prepared samples of Au/mica.

Additionally, we found at nanometer scale that cis-stilbene forms clusters on HOPG as well, similarly to trans-stilbene. A gradual increase in the amount of cis-stilbene enlarges the clusters until they coalesce in an amorphous phase. In contrast, the molecule-substrate interactions of cis-stilbene on Au/mica are weaker than on HOPG and do not form crystal nuclei centers or stable nanostructures. Instead, increasing cis-stilbene molecular concentration on $\mathrm{Au} /$ mica leads to a sort of liquid film.

Keywords: $c i s$-stilbene, trans-stilbene, HOPG, Au/mica, AFM.

\section{INTRODUCCIÓN}

El estudio y comprensión de fenómenos a escala nanométrica han tenido un gran auge en las últimas décadas, principalmente gracias a los avances tecnológicos y al desarrollo de instrumental que permite estudiar y manipular materiales a nivel atómico y molecular. Muchos de los nanomateriales sintéticos diseñados recientemente están inspirados en la naturaleza [1]. De hecho, la naturaleza hace uso de la nanotecnología en un sin fin de funciones: la replicación de ADN, el nanotransporte a través de membranas celulares y uso de máquinas del tamaño de una molécula para la generación de adenosín trifosfato (ATP), son algunos ejemplos [2]. En los últimos años, la creciente demanda de productos de bajo impacto ambiental ha incentivado la optimización del uso de recursos naturales renovables, de tal modo que sirvan para reemplazar a otros menos sustentables. Por ejemplo, derivados vegetales están siendo estudiados con el fin de reemplazar otros de origen sintético [3-ㅡ]. Actualmente la nanotecnología se presenta como una herramienta alternativa para descubrir propiedades y fenómenos nuevos, que puedan contribuir a un uso más eficiente de la materia (menos cantidad o miniaturización, menor consumo energético, mejor performance) y a la exploración de nuevas tecnologías.

Varias moléculas orgánicas de origen vegetal (ya sea extractos o derivados de éstos) presentan una estructura química capaz de experimentar cambios estructurales u otros tipos de isomerización ante la influencia de factores externos $[4, \underline{7}, 8]$. Algunas también presentan propiedades de utilidad en la preparación de nanoestructuras y máquinas moleculares. Sin embargo, aún no han sido exploradas en esta dirección y poco se conoce acerca de su comportamiento en superficies, sus interacciones con sustratos sólidos e intermoleculares, o su movilidad cuando están depositadas en un sólido. Uno de los ejemplos de interés es el estilbeno, así como también sus formas isoméricas. Estas moléculas en general son bien conocidas por sus aplicaciones en farmacia, medicina y en la industria alimenticia [9]. Si bien mucho se sabe acerca de sus propiedades físicas y químicas cuando se encuentran en solución líquida o fase gaseosa, su potencial como unidades para la fabricación de nanoestructuras radica en controlar su comportamiento en estado sólido $[10, \underline{11}]$. Por ello, el enfoque abordado en este trabajo es integrar moléculas de estilbeno en un medio sólido, más precisamente inmovilizarlas o depositarlas sobre superficies de características conocidas. De hecho, la inmovilización de moléculas sobre un sustrato es un paso clave en nanoingeniería [08] ya que (i) permite ubicarlas en el lugar deseado, (ii) ofrece la posibilidad de mantenerlas apartadas unas de otras en caso de ser necesario, (iii) evita colisiones indeseadas con otras moléculas del entorno y (iv) limita el movimiento Browniano y los grados de libertad a los que está sujeto cualquier ente de dimensiones nanométricas. Varios trabajos recientes muestran que trans-estilbeno puede formar estructuras ordenadas sobre algunos sustratos cristalinos. Sin embargo, las observaciones por microscopía de fuerza atómica (AFM) o microscopía túnel de barrido (STM) se llevaron a cabo siempre en ultra alto vacío y a bajas temperaturas [12-14]. Pero si estamos interesados en conocer o desarrollar materiales que puedan aplicarse a nuevas tecnologías es importante caracterizar el sistema estilbeno/sustrato en condiciones ambientales. 


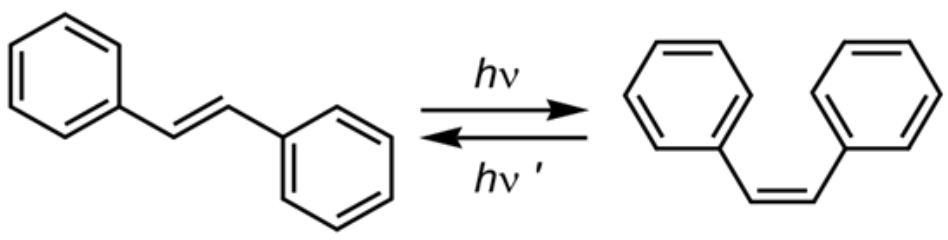

Figura 1: Estructura química de los isómeros trans- (izquierda) y cis-estilbeno (derecha).

Por todo lo antes expuesto, este trabajo presenta los primeros resultados obtenidos al estudiar formas isoméricas de estilbeno sobre superficies cristalinas de oro y grafito. Los isómeros trans- y cis-estilbeno (ver Fig. 1) se presentan como un sólido y un líquido a temperatura ambiente, respectivamente. Los sistemas se analizan mediante microscopía de fuerza atómica (AFM) y microscopía óptica en los casos posibles en condiciones atmosféricas, con el objetivo de explorar y comparar sus propiedades cuando son depositadas en superficie.

\section{MATERIALES Y MÉTODOS}

Se utilizaron sustratos de grafito pirolítico altamente orientado (HOPG) y oro soportado en mica (Au/mica). Se empleó HOPG marca SPI Supplies - Grado 1, clivado inmediatamente antes de preparar las muestras. Los sustratos de Au/mica fueron suministrados por el Zernike Institute for Advanced Materials (Universidad de Groningen, Países Bajos). Para su preparación, la mica fue clivada y pre-calentada a $640 \mathrm{~K}$ por 12 horas en una cámara de vacío con presión base de $10^{-7}$ Torr, a los fines de expulsar impurezas medioambientales y humedad. Seguidamente, se depositaron $150 \mathrm{~nm}$ de Au al $99.99 \%$ manteniendo la mica a $640 \mathrm{~K}$. Finalmente, el sustrato se enfrió hasta llegar a temperatura ambiente en el transcurso de 8 horas. Mediante este método se obtuvieron terrazas atómicamente planas de $\mathrm{Au}(111)$, tal como se ha descrito en otras oportunidades [15]. La elección de dichos sustratos se debe a que sus propiedades son bien conocidas y resultan convenientes para ser analizados por AFM [16].

Para este estudio se prepararon muestras de dos isómeros de estilbeno (trans-1,2-Diphenylethylene, CAS 103-30-0, o cis-1,2-Diphenylethylene, CAS 645-49-8) en solución de etanol absoluto. Se prepararon soluciones de varias concentraciones a partir de estos isómeros. Se depositaron gotas de volumen conocido de cada solución sobre HOPG y Au/mica, dejando evaporar el solvente en condiciones atmosféricas. Muestras así preparadas fueron analizadas por AFM y microscopía óptica.

Las medidas de AFM se realizaron con un equipo comercial de marca Nanotec Electrónica S.L. (Madrid, España), en aire y temperatura ambiente, operando en modo contacto intermitente. Se utilizaron "cantilevers" Olympus RC800PSA y Budget Sensors Multi75Al de silicio (los primeros nitrurados) recubiertos con $\mathrm{Au} / \mathrm{Cr}$ y $\mathrm{Al}$, respectivamente, con frecuencias de resonancia entre $70-90 \mathrm{kHz}$, fuerzas nominales entre 0.4 $2.7 \mathrm{~N} / \mathrm{m}$ y radios de curvatura menores a $20 \mathrm{~nm}$. Las imágenes se adquirieron y procesaron con el "software" libre WS $\times$ M [17].

Además, se utilizó un microscopio óptico de alta resolución de marca Nanotec Electrónica S.L. (Madrid, España), de movimiento axial 3D y cuyas lentes tienen tecnología CMOS. Lleva acoplada una cámara CCD que permite observar el movimiento del "cantilever" sobre la muestra en tiempo real simultáneamente con la adquisición de las imágenes AFM, y obtener mediante el programa WS $\times$ M fotografías a escalas micrométricas de las estructuras formadas sobre las muestras a lo largo de los experimentos.

\section{RESULTADOS Y DISCUSIÓN}

La Fig. 2 muestra imágenes AFM de superficies de HOPG recubiertas con cantidades crecientes de transestilbeno. Estas cantidades crecientes se obtuvieron preparando muestras a partir de soluciones cada vez más concentradas. La superficie de HOPG sin moléculas muestra terrazas monoatómicas, escalones y defectos característicos de este tipo de sustratos (imagen no mostrada aquí). La interacción de la solución más diluida de trans-estilbeno con HOPG origina la formación de nanopartículas, que se ven como puntos brillantes ubicados preferentemente en los defectos del sustrato (Fig. 2(a)). En la imagen de fase (Fig. 2(d)) se observa que las nanopartículas tienen un contraste muy diferente al del sustrato, lo cual sugiere que son de un material distinto, que se atribuye a trans-estilbeno. Estas partículas se mantienen estables durante sucesivos escaneos para la adquisición de imágenes. Un aumento en la cantidad de moléculas da lugar a la formación de aglomerados que crecen en forma de islas (Figs. 2(b) y 2(e)). La Fig. 2(c) presenta un estado de saturación, donde el HOPG está totalmente cubierto por islas de moléculas. La falta de contraste significativo en la correspondien- 
te imagen de fase (Fig. 2(f)) permite inferir que todo el HOPG está cubierto.

La observación de esta muestra al microscopio óptico revela la formación de cristales tridimensionales de trans-estilbeno que comienzan a distinguirse a simple vista. Estos cristales presentan una morfología mayoritariamente pseudo-romboide (Fig. 3). Se ha intentado analizar la muestra mediante microscopía electrónica para estudiar estadios intermedios en la formación de los cristales de trans-estilbeno a escala mesoscópica, pero los mismos se degradan por efecto de las condiciones experimentales de adquisición, dificultando las mediciones.

En la Fig. 4 se muestran imágenes AFM de Au/mica sin moléculas de estilbeno (Fig. 4(a)) y recubierto con cantidades crecientes de trans-estilbeno (Figs. 4(b-c)), con concentraciones comparables a los casos mostrados en las Figs. 2(b-c). De manera similar al estudio realizado sobre HOPG, estas cantidades crecientes de moléculas fueron depositadas preparando muestras a partir de soluciones de trans-estilbeno cada vez más concentradas. En la parte inferior (Figs. 4(d-f)) se presentan las imágenes de fase correspondientes a cada una de las de topografía. El sustrato de Au/mica sin estilbeno muestra zonas cristalinas, donde se observan triángulos con terrazas y escalones típicos de Au con una orientación (111). La Fig. 4(b) muestra una superficie recubierta con "clusters" de trans-estilbeno. La correspondiente imagen de fase (Fig. 4(e)) revela que toda la superficie de Au/mica está cubierta y decorada con estas formaciones.
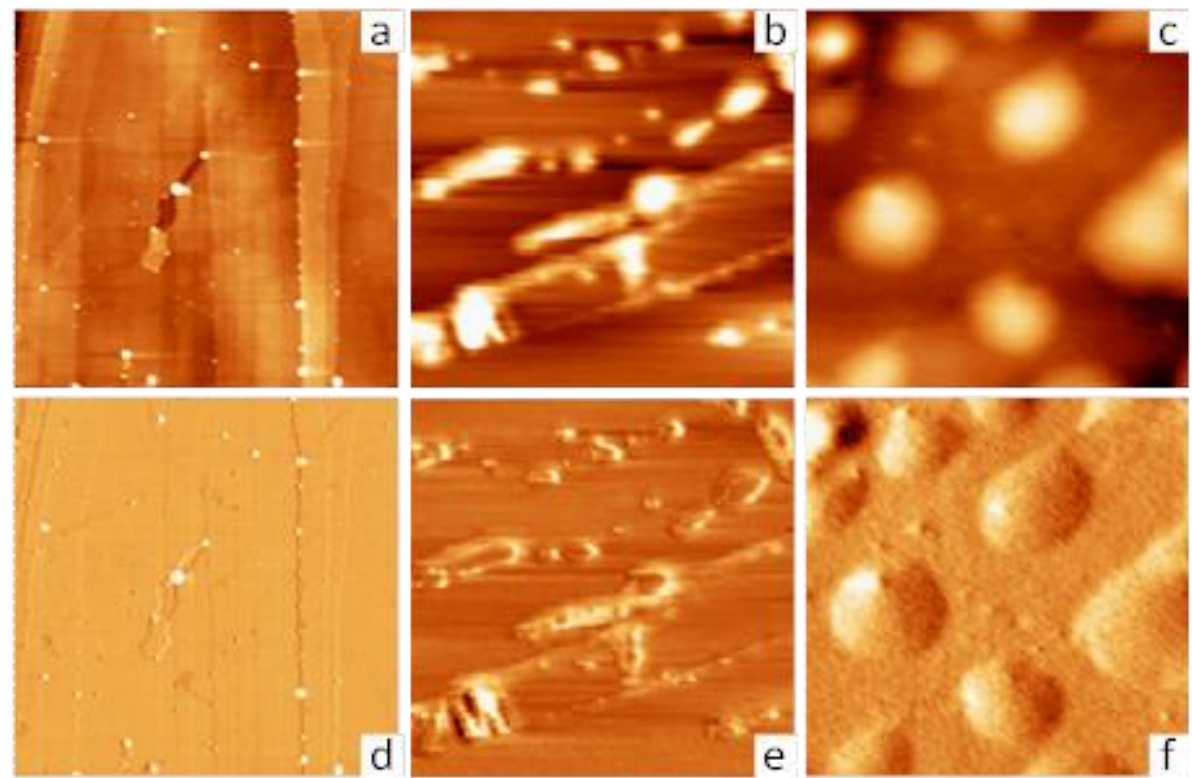

Figura 2: Imágenes AFM $(3000 \mathrm{~nm} \times 3000 \mathrm{~nm})$ de trans-estilbeno sobre HOPG. En el panel superior e inferior se muestran imágenes de topografía y fase, respectivamente. De izquierda a derecha, recubrimientos incrementales de transestilbeno sobre HOPG.
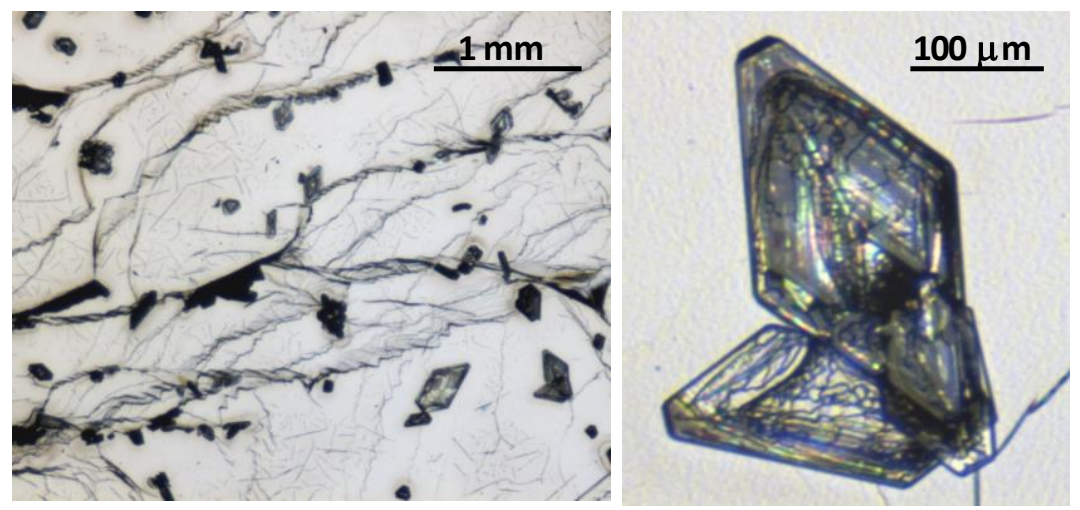

Figura 3: Imágenes de trans-estilbeno sobre HOPG, tomadas mediante microscopio óptico. Izquierda: se observa la formación de pequeños cristales que comienzan a distinguirse a simple vista. Derecha: detalle de un grupo de cristales. 
Un incremento en la concentración de moléculas, da origen a estadios como el que ilustra la Fig. 4(c). En este caso el Au/mica se recubrió a partir de una solución de concentración idéntica a la muestra sobre HOPG de la Fig. 2(c). Si bien aquí mediante AFM no se distingue un ordenamiento significativo aún cuando se ve toda la superficie cubierta con material, la inspección de la misma mediante microscopio óptico (Fig. $5(a-b))$ revela el crecimiento de cristales de trans-estilbeno en forma dendrítica, siguiendo estructuras con un eje central ramificado. El eje central de los cristales tiene una orientación más bien azarosa, con ramificaciones mayoritariamente en ángulos a $90^{\circ}$. Mientras la Fig. 5(a) muestra una vista general de la muestra, la Fig. 5(b) presenta en detalle los cristales, según se ven al microscopio óptico. La Fig. 5(c) muestra una imagen AFM donde se observa la formación incipiente de estructuras dendríticas que inician patrones de ramificación tendientes a orientarse en un ángulo recto.

La Fig. 6 ejemplifica las formaciones que generan las moléculas de cis-estilbeno cuando se depositan sobre HOPG. El cis-estilbeno se agrupa formando nanopartículas e islas ubicadas preferentemente en los escalones del sustrato. Es de notar que a escala nanométrica las formaciones del isómero cis son similares a las del trans (ver Figs. 2(b-c)), a pesar de que el primero es un líquido y el segundo un sólido a escala macroscópica.
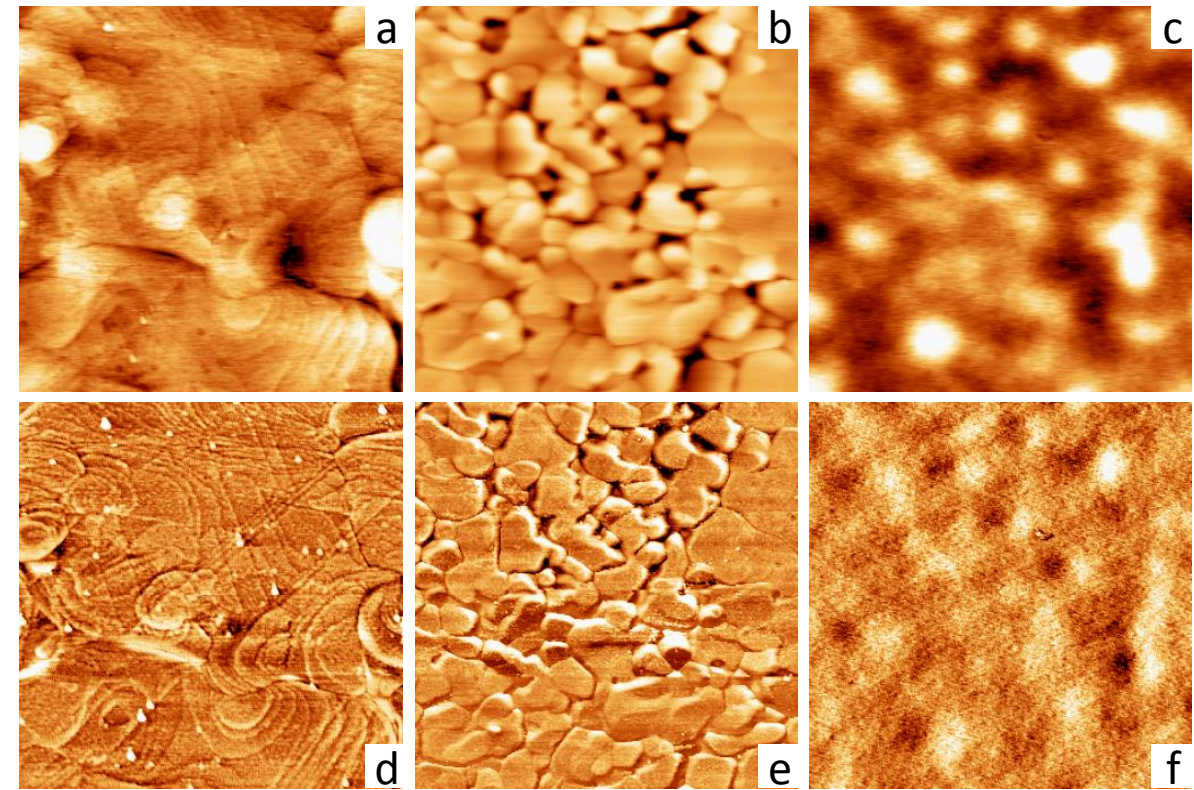

Figura 4: Imágenes AFM $(3000 \mathrm{~nm} \times 3000 \mathrm{~nm})$ de trans-estilbeno sobre Au/mica, En el panel superior e inferior se muestran imágenes de topografía y fase, respectivamente. De izquierda a derecha, (a) Au/mica sin trans-estilbeno, (b-c) $\mathrm{Au} /$ mica con recubrimientos incrementales de trans-estilbeno.
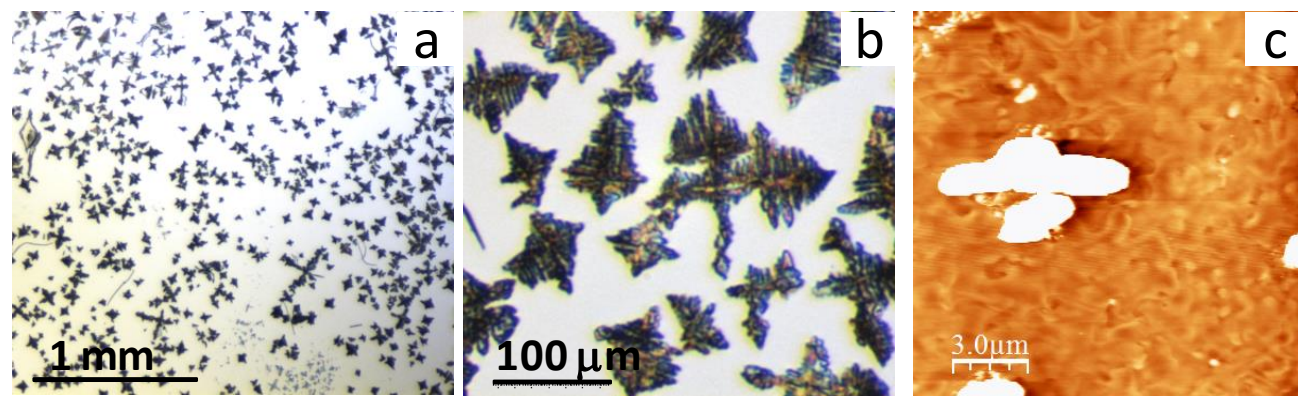

Figura 5: (a-b) Imágenes de trans-estilbeno sobre Au/mica, tomadas con microscopio óptico. (c) Imagen AFM (12000 $\mathrm{nm} \times 12000 \mathrm{~nm}$ ) donde se observa la formación incipiente de estructuras dendríticas. 


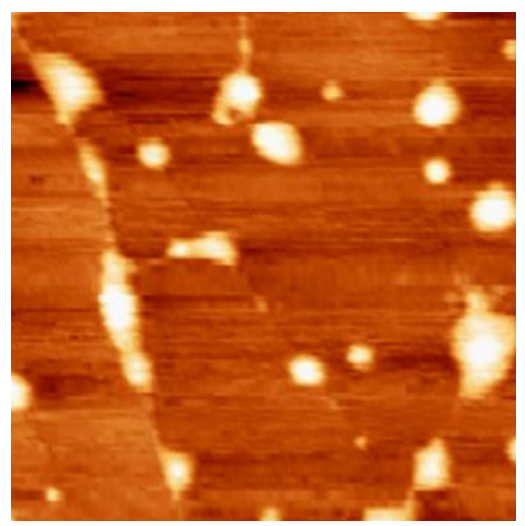

Figura 6: Imagen AFM $(3000 \mathrm{~nm} \times 3000 \mathrm{~nm})$ de cis-estilbeno sobre HOPG.

La Fig. 7 muestra imágenes AFM de cis-estilbeno sobre Au/mica, correspondientes a muestras preparadas a partir de soluciones con concentraciones equivalentes a las utilizadas en el caso de trans-estilbeno. A diferencia del isómero trans, si bien se observan "clusters" de moléculas, ya no presentan bordes de grano bien definidos (comparar Figs. 4(b) y 7(a)). Un paulatino aumento en la concertación de cis-estilbeno sobre $\mathrm{Au} /$ mica deriva en la coalescencia de dichos "clusters" en una película delgada líquida amorfa. La Fig. 7(b) corresponde a la topografía de una "gota" de cis-estilbeno. Muestras preparadas a partir de soluciones de menor concentración tampoco dieron lugar a la formación de centros de nucleación estables. Curiosamente, las imágenes de fase permiten distinguir partículas de un material diferente, de mayor dureza (ver partículas brillantes en la Fig. 7(e)). Un detalle de estas formaciones se muestra en las Figs. 7(c) y 7(f). Es posible que se deban a nanopartículas de trans-estilbeno, formadas por la isomerización de cis-estilbeno (Fig. 1), que actúan como centros de nucleación para la formación de estructuras de dureza diferente y por ende registrables mediante AFM. De todos modos, los datos experimentales aquí mostradas no son suficientes para confirmar esta hipótesis.
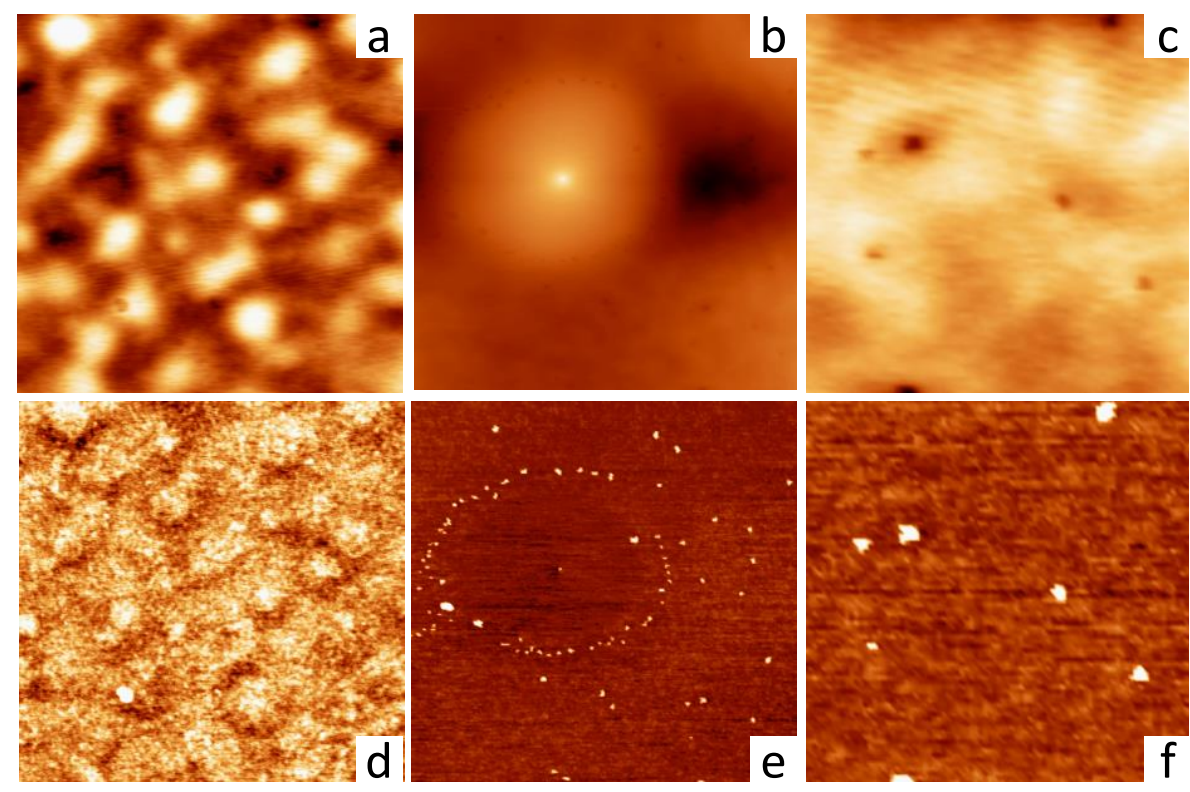

Figura 7: Imágenes AFM de cis-estilbeno sobre Au/mica. En el panel superior e inferior se muestran imágenes de topografía y fase, respectivamente. (a) $(3000 \mathrm{~nm} \times 3000 \mathrm{~nm})$ con un recubrimiento equivalente al de la Fig. 4(b), (b) (15000 $\mathrm{nm} \times 15000 \mathrm{~nm})$ con un recubrimiento equivalente al de la Fig. 4(c), (c) $(5000 \mathrm{~nm} \times 5000 \mathrm{~nm})$ ampliación de (b).

\section{CONCLUSIONES}

El presente trabajo muestra que la inmovilización de trans-estilbeno sobre una superficie, modifica el proceso de cristalización del recubrimiento orgánico, cuya morfología depende del tipo de sustrato. En el caso de trans-estilbeno sobre HOPG, se obtienen cristales de forma pseudo-romboide, los que se originan a partir de nanopartículas o centros de nucleación ubicados preferentemente en los defectos de la superficie del sustrato. 
La interacción entre moléculas de trans-estilbeno es mucho más fuerte que la interacción molécula-sustrato y un paulatino incremento en la cantidad de trans-estilbeno en la superficie se traduce en un crecimiento de granos que generan la formación de cristales macroscópicos. Sin embargo, trans-estilbeno preparado de igual manera pero sobre muestras de Au/mica, resulta en superficies cubiertas preferentemente por formaciones de estructuras dendríticas con ramificaciones con ángulos a $90^{\circ}$. Para muestras preparadas según se describió, la concentración de la solución de partida es un factor que puede afectar la estructura final del recubrimiento, ya que un cambio en la concentración de la solución incide en el número de centros de nucleación a partir de los cuales pueden crecer los cristales. Por ello, al comparar muestras de trans-estilbeno sobre sustratos distintos, es importante prepararlas a partir de soluciones con concentraciones idénticas.

Por otra parte, cis-estilbeno que se presenta como un líquido a escala macroscópica y condiciones atmosféricas, a escala nanométrica forma estructuras similares a las de su isómero sólido, el trans-estilbeno, sobre HOPG. En cambio, sobre Au/mica la interacción molécula-sustrato es más lábil y ya no resulta posible observar centros de nucleación incipientes o nanoestructuras de cis-estilbeno estables como cuando se deposita sobre HOPG o como en el caso de trans-estilbeno sobre Au/mica.

Finalmente, cabe mencionar que otro factor que puede influir de manera significativa en la formación de estructuras de cis- y trans-estibeno sobre Au/mica o HOPG es el solvente, ya que el método de preparación de películas delgadas aquí utilizado implica un proceso dinámico de evaporación del mismo. Como todas las muestras fueron preparadas utilizando un único solvente, el estudio de este factor escapa a los alcances del presente trabajo.

\section{AGRADECIMIENTOS}

Este trabajo se realizó en el marco del proyecto PICT 20101637 (ANCyPT-FONCyT), con apoyo de la Universidad Tecnológica Nacional y del Instituto de Física del Litoral (CONICET-UNL). Además, se agradece la colaboración de la Profesora Dra. P. Rudolf, del Zernike Institute for Advanced Materials (Países Bajos), por donar los sustratos de Au/mica.

\section{BIBLIOGRAFÍA}

[1] BHUSHAN (Ed), Handbook of Nanotechnology. Springer-Verlag Berlin Heidelberg, Alemania, 2004.

[2] SCHLIWA, M. (Ed), Molecular Motors. Wiley-VCH, Weinheim, 2003.

[3] MOHANTY, A.K., MISRA, M., DRZAL, L.T. (Eds), Natural Fibers, Biopolymers and Biocomposites. CRC Press - Taylor \& Francis Group, Boca Raton, 2005.

[4] HUBBE, M.A., ROJAS, O.J., LUCIA, L.A., et al., "Cellulosic nanocomposites: a review”, Bioresources v. 3, n. 3, pp. 929-980, 2008.

[5] SATYANARAYANA, K. G., ARIZAGA, G.G.C., WYPYCH, F., "Biodegradable composites based on lignocellulosic fibers-An overview”, Progress in Polymer Science, v. 34 pp. 982-1021, Enero 2009.

[6] SEKI, Y. "Innovative multifunctional siloxane treatment of jute fiber surface and its effect on the mechanical properties of jute/thermoset composites", Materials Science and Engineering: A, v. 508, n. 1-2 pp. 247-252, mayo 2009.

[7] SCHMIDT, P.M, HORN, K., HUGO DIL, J., et al., "Conformational isomers of stilbene on Si(1 000$)$ ”, Surface Science, v. 601, pp. 1775-1780, Abril 2007.

[8] KOTTAS, G.S., CLARKE, L.I., HORINEK, D., et al., “Artificial Molecular Rotors”, Chemistry Review, v. 105, n. 4, pp. 1281-1376, Abril 2005.

[9] LIKHTENSHTEIN, G., Stilbenes: applications in chemistry, life sciences and materials science. WileyVCH Verlag GmbH \& Co. KGaA, Weinheim, 2010.

[10] BALZANI, V., CREDI, A., VENTURINI, M., "Molecular devices and machines", Nano Today v. 2, n. 2, pp. 18-25, Abril 2007.

[11] HENRIKSSON, M., BERGLUND, L.A., ISAKSSON, P., LINDSTROM, T., NISHINO, T., "Cellulose nanopaper structures of high toughness". Biomacromolecules, v. 9, n. 6, pp. 1579-1585, Mayo 2006.

[12] TSAI , C.-S., SU , C., WANG, J.-K, et al." STM Study of trans-stilbene self-organized on the $\mathrm{Ag} / \mathrm{Ge}(111)-(\sqrt{3} \times \sqrt{3}) \mathrm{R} 30^{\circ}$ surface”, Langmuir, v. 19, n. 3, pp. 822-829, Enero 2003.

[13] RIEDEL, D., CRANNEY, M., MARTIN, M., et al., "Surface-isomerization dynamics of trans-stilbene molecules adsorbed on Si(100)-2×1”, J. of Am. Chem. Soc, v. 131, n. 15, pp. 5414-5423, Marzo 2009. 
[14] SU, C., LEE, L.-X., CHEN, C.-F., "Scanning tunneling microscopy observation of stilbene derivative self-assembled on HOPG”, Synthetic Metals, v. 137, n. 1-3, pp. 865-866, Abril 2003.

[15] MENDOZA, S.M., ARFAOUI, I.E., ZANARINI, S., et al., "Improvements in the characterization of the crystalline structure of acid-terminated alkanethiol self-assembled monolayers on Au(111)", Langmuir , v. 23, n. 2, pp. 582-588, Nov. 2007.

[16] MENDOZA, S.M., Exploiting molecular machines on surfaces, Tesis de D.Sc., RUG, Groningen, Países Bajos, 2007.

[17] HORCAS, I., FERNÁNDEZ, R., GÓMEZ-RODRÍGUEZ, J.M., et al., "WSXM: A software for scanning probe microscopy and a tool for nanotechnology”, Rev. Sci. Instrum., v. 78, pp 013705/1-8, Enero 2007. 